\title{
Free-standing and ultrathin inorganic light-emitting diode array
}

\author{
Youngbin Tchoe ${ }^{1}$, Kunook Chung ${ }^{1}$, Keundong Lee', Janghyun Jo ${ }^{3}$, Kyungmin Chung ${ }^{2}$, Jerome K. Hyun², \\ Miyoung $\mathrm{Kim}^{3}$ and Gyu-Chul Yi ${ }^{1}$
}

\begin{abstract}
We report on the fabrication and characteristics of an individually addressable gallium nitride (GaN) microdisk lightemitting diode (LED) array in free-standing and ultrathin form. A high-quality GaN microdisk array with $n$-GaN, InGaN/ GaN quantum wells and $p$-GaN layers was epitaxially grown on graphene microdots patterned on $\mathrm{SiO}_{2} / \mathrm{Si}$ substrates. Due to the weak attachment of the graphene microdots to the growth substrate, a microdisk array coated with a polyimide layer was easily separated from the substrate using mechanical or chemical methods to form an ultrathin free-standing film. Individually addressable microdisk LEDs were created by forming thin metal contacts on the $p$-GaN and $n$-GaN surfaces in a crossbar configuration. Each microdisk LED that comprised an ultrahigh resolution array of 2500 pixels per inch was found to be uniquely addressable. The devices in free-standing form exhibited stable electrical and optoelectronic characteristics under extreme bending conditions and continuous operation mode despite the absence of a heat dissipating substrate. These results present promising approaches for the fabrication of high-quality inorganic semiconductor devices for ultrahigh resolution and high-performance flexible applications.
\end{abstract}

\section{Introduction}

Inorganic microlight-emitting diode (micro-LED) arrays are emerging as one of the most promising light emitters for next-generation display technologies and ultrahigh resolution optogenetic light source arrays ${ }^{1-5}$. An inorganic micro-LED can exhibit all of the important characteristics of an organic LED, including fast response time and a high contrast ratio, but with significantly improved resolution, brightness, efficiency, and lifetime due to its increased carrier mobilities, radiative recombination rates, and long-term stability ${ }^{6-10}$. In free-standing and ultrathin form, the applicability of micro-LEDs can be further expanded to include various wearable, medical, and implantable devices ${ }^{5,11-14}$, which require conformal contact on human skin or organs with minimal discomfort

\footnotetext{
Correspondence: Gyu-Chul Yi (gcyi@snu.ac.kr)

${ }^{1}$ Department of Physics and Astronomy, Institute of Applied Physics, and Research Institute of Advanced Materials, Seoul National University, Seoul 08826, Korea

${ }^{2}$ Department of Chemistry and Nanoscience, Ewha Womans University, Seoul 03760, Korea

Full list of author information is available at the end of the article.
}

and stress. By creating an array of free-standing inorganic microstructure devices that are orders of magnitude smaller than the bending radius, mechanical flexibility can be accommodated ${ }^{15,16}$. However, the inherent rigidity of inorganic materials and difficulty separating inorganic thin films from their single crystal growth substrates represent substantial challenges to the fabrication of freestanding and ultrathin inorganic LEDs. Herein, we report on the fabrication and electroluminescence (EL) characteristics of a flexible and individually addressable gallium nitride $(\mathrm{GaN})$ microdisk LED array in free-standing and ultrathin form, enabled by position-controlled graphene microdots that act as microdisk growth and release sites.

Substantial effort has been made to produce flexible inorganic LED arrays due to the many advantages mentioned above. One of the most successful demonstrations of flexible inorganic LED arrays is the laser liftoff of inorganic thin film LEDs grown on sapphire substrates and assembled on flexible substrates ${ }^{17,18}$. This approach provides direct integration of highly optimized thin film

\section{(c) The Author(s) 2019}

(c) (i) Open Access This article is licensed under a Creative Commons Attribution 4.0 International License, which permits use, sharing, adaptation, distribution and reproduction cc) in any medium or format, as long as you give appropriate credit to the original author(s) and the source, provide a link to the Creative Commons license, and indicate if changes were made. The images or other third party material in this article are included in the article's Creative Commons license, unless indicated otherwise in a credit line to the material. If material is not included in the article's Creative Commons license and your intended use is not permitted by statutory regulation or exceeds the permitted use, you will need to obtain permission directly from the copyright holder. To view a copy of this license, visit http://creativecommons.org/licenses/by/4.0/. 
LEDs onto a flexible platform. Moreover, the nanostructured LED-based approach of fabricating flexible inorganic LED arrays has also been extensively studied due to the recent high interest in micro-LED arrays that require orders of magnitude smaller LEDs compared to conventional thin film LEDs. Nanowires could be easily lifted off from the substrates due to their nanoscale contact area with the substrate, and discrete nanostructure LEDs embedded in flexible polymer layers showed reliable operation under flexible conditions ${ }^{19}$. More recently, semiconductor nanostructure growth on graphene films and their application to flexible LEDs were demonstrated as a method to prepare large area, transferrable LEDs without the need for single crystal substrates ${ }^{20,21}$. However, a crucial step toward the realization of highresolution flexible micro-LED arrays is the demonstration of individual operation of a single nanostructure LED in a flexible array. In this work, we demonstrate an individually addressable GaN microdisk LED in a freestanding and ultrathin flexible array. This free-standing and ultrathin light source with a high spatial resolution could potentially be used for many biomedical applications.

\section{Materials and methods}

\section{Growth of GaN microdisk arrays on graphene dots}

For the epitaxial lateral overgrowth of the GaN microdisks, continuous graphene films were patterned into microdot arrays by photolithography and $\mathrm{O}_{2}$ plasma dry etching. Initially, $c$-axis aligned zinc oxide $(\mathrm{ZnO})$ nanowalls were grown on the graphene microdots using metal organic vapor phase epitaxy (MOVPE). GaN microdisks were then formed using epitaxial lateral overgrowth on the $\mathrm{ZnO}$-covered graphene dots using a pulsed-mode MOVPE technique. Additional layers of $\mathrm{Mg}$-doped $p$ $\mathrm{GaN}, \quad u-\mathrm{In}_{x} \mathrm{Ga}_{1-x} \mathrm{~N} / \mathrm{u}-\mathrm{GaN}$ multiple quantum wells (MQWs), and Si-doped $n$-GaN layers were subsequently grown on the microdisks.

\section{Preparation of ultrathin and flexible free-standing GaN microdisk LED structure}

Large-area grown GaN microdisk arrays on graphene microdots were used as a starting material for the fabrication of free-standing, ultrathin LED arrays. The first step was to transfer parts of the GaN microdisk arrays from the original substrate to a foreign substrate. To transfer the $\mathrm{GaN}$ microdisks while preserving the order and regularity of the array, a polyimide (PI) layer was spin coated onto the GaN microdisk array. The GaN microdisk array was then lifted off by mechanical peel off or by wet chemical etching of the underlying sacrificial layer using a buffered oxide etchant (BOE). Then, under an optical microscope, the GaN microdisk array in PI was divided into smaller pieces. After transferring a small piece of the
GaN microdisk array onto a foreign $\mathrm{SiO}_{2} / \mathrm{Si}$ substrate, a 2nd PI layer was spin coated onto the substrate. Then, the $\mathrm{SiO}_{2}$ layer was removed by a $\mathrm{BOE}$, completing the formation of an ultrathin and free-standing layer composed of a GaN microdisk array embedded in PI.

Before metallization, the surfaces of $p-\mathrm{GaN}$ and graphene microdots were exposed by selectively etching the PI layers covering the top and bottom surfaces of the GaN microdisks. Polymethyle methacrylate (PMMA) layers were first coated on the ultrathin PI layer covering the microdisk array, and a 3- $\mu$ m-diameter hole was patterned at the position of each $\mathrm{GaN}$ microdisk. Then, an oxygen plasma treatment was used to selectively etch the PI layer through the patterned PMMA mask. The selective etching of the PI layer was performed on both the top and bottom surfaces of the GaN microdisk LED array, thereby exposing the surface of the $p$-GaN and graphene microdots.

\section{Fabrication of individually addressable GaN microdisk LED arrays with SWCNT-embedded microelectrodes}

Single-walled carbon nanotube (SWCNT)/Ni/Au and SWCNT/Ti/Au multiple electrode lines were formed on the top and bottom surfaces of the GaN microdisk arrays in a crossbar configuration. The SWCNT-embedded metal electrodes were formed by dispersing SWCNTs onto the ultrathin layer first and depositing the microelectrodes afterwards. Then, the SWCNTs that were not covered by the metal electrodes were etched. After metallization, the LEDs were postannealed in $\mathrm{N}_{2}$ for $5 \mathrm{~min}$ at $300^{\circ} \mathrm{C}$.

\section{LED characterization}

An optical microscope (Carl Zeiss Co. Axioskop 2 MAT) was used for imaging the probing and EL emission collection. An electrical source meter (Keithley 2400) was used to measure current-voltage (I-V) curves and to apply voltages or currents to the LEDs. The EL spectra were measured using a monochromator (Dongwoo Optron Co. DM150i) and a detection system equipped with a charge-coupled device (Andor Inc. DU401A). The resolution of the EL spectrum was $1 \mathrm{~nm}$, and the typical scan range was between 300 and $800 \mathrm{~nm}$.

\section{Computational modeling of the current spreading and light output characteristics}

The current spreading characteristics of the microdisk LED were modeled using a COMSOL Multiphysics 4.3b semiconductor module. The donor and acceptor concentrations of $n$ - and $p$-GaN that we used in the calculations were $5.0 \times 10^{18}$ and $2.0 \times 10^{17} \mathrm{~cm}^{-3}$, respectively, while the intrinsic carrier concentration was set to $1.0 \times$ $10^{17} \mathrm{~cm}^{-3}$. The band gap, electron mobility, and hole mobility values of GaN we used were $3.39 \mathrm{eV}, 440 \mathrm{~cm}^{2} / \mathrm{V} \cdot \mathrm{s}$, and $200 \mathrm{~cm}^{2} / \mathrm{V} \cdot \mathrm{s}$, respectively. 


\section{Results and discussion}

\section{Preparation of free-standing and ultrathin GaN microdisk arrays}

The basic strategy for the fabrication of an ultrathin and free-standing GaN microdisk array embedded in polyimide film (Fig. 1a) is as follows. Each GaN microdisk array element, composed of a $\mathrm{u}-\mathrm{In}_{x} \mathrm{Ga}_{1-x} \mathrm{~N} / \mathrm{GaN}$ multiple quantum wells in between $n-\mathrm{GaN}$ and $p$-GaN layers, was prepared by epitaxial growth using selective-area MOVPE on a $\mathrm{ZnO}$-coated patterned graphene microdot supported by a $\mathrm{SiO}_{2} / \mathrm{Si}$ substrate ${ }^{22}$. The graphene microdot array was designed with a diameter and period of 3 and $10 \mu \mathrm{m}$, respectively. The weak bonding of graphene microdots to $\mathrm{SiO}_{2} / \mathrm{Si}$ allows the microdisks to be easily separated from the substrate by mechanical peel off to form a freestanding film. An alternative liftoff method uses a wetchemical etch approach on the underlying amorphous sacrificial layers. After the growth of $\mathrm{GaN}$ microdisk arrays, we used a twofold PI transfer technique to pick up a small subarray of $\mathrm{GaN}$ microdisks from a large microdisk array and embed them into an ultrathin polymer layer. This enabled the fabrication of numerous flexible microdisk LED arrays from a single growth of a large-area GaN microdisk array (see Fig. S1). The lifted-off layer, with sizes of a few squared centimeters, was then divided into smaller arrays with sizes of $200 \mu \mathrm{m} \times 200 \mu \mathrm{m}$. Each

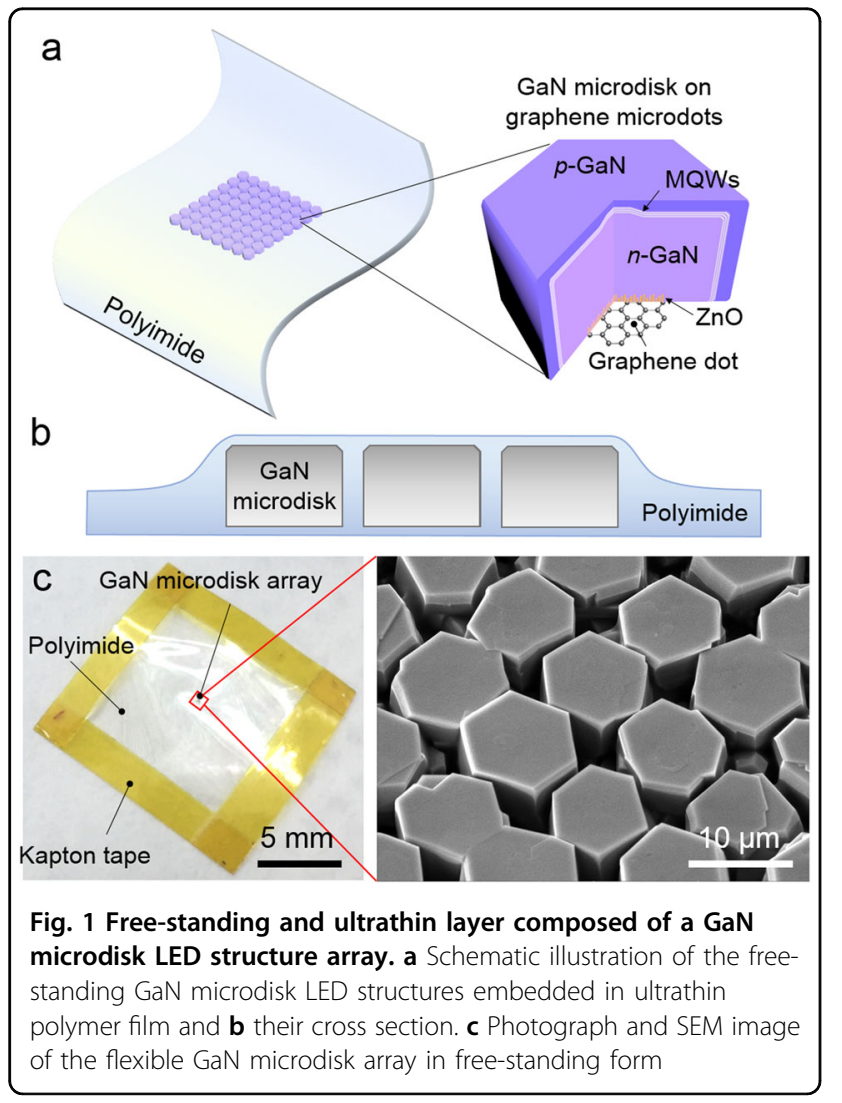

subarray was transferred onto a foreign carrier substrate. A second PI layer was spin coated on the array and subsequently peeled off from the carrier substrate. As shown in the cross-sectional schematics of the lifted-off layer in Fig. 1b, the thickness of the PI layers near the GaN microdisk arrays were $8 \mu \mathrm{m}$, while the PI layers outside the microdisk arrays were $3 \mu \mathrm{m}$ in thickness. Figure 1c shows the photograph and the corresponding scanning electron microscopy (SEM) image of a typical freestanding, ultrathin layer containing a microdisk array. The structural integrity and order of the microdisk array were remarkably well preserved despite the absence of a solid support. In addition, the SEM image shows that some microdisk LEDs touched or merged with each other after the $1-\mu \mathrm{m}$-thick $p$-GaN coating. However, considering the fact that the $n-\mathrm{GaN}$ microdisks were smaller in size and clearly separated, and the $p$-GaN layers were highly resistive, the touching or merging between $p$-GaN layers did not affect the individual operation of the LEDs, which is demonstrated later in the paper.

\section{Device fabrication of the individually addressable GaN microdisk LED array}

To enable each micro-LED to be individually addressable, metal layers were deposited on the top and bottom surfaces of the free-standing microdisk array. Figure 2 shows the schematic and SEM images for each metallization process. The first step was to expose the top $p-\mathrm{GaN}$ and bottom $n-\mathrm{GaN}$ surfaces of the microdisks by removing the polyimide layer with electron beam lithography and oxygen plasma etching (Fig. 2a). Circular microdots displaying the exposed $p$ - and $n$-GaN surfaces on the top and bottom of the microdisks are clearly shown in the SEM images. Second, SWCNTs were deposited on the top and bottom surfaces of the polyimide, including the exposed regions, to act as flexible and stretchable backbones to the electrode (Fig. 2b). Thereafter, thin Ni/Au $(10 / 100 \mathrm{~nm})$ and $\mathrm{Ti} / \mathrm{Au}(10 / 100 \mathrm{~nm})$ metal strips were deposited in a crossbar configuration to one another on the top $p$-GaN and bottom $n$-GaN surfaces, respectively. Finally, the SWCNTs that were not covered by the metal strips were removed by $\mathrm{O}_{2}$ reactive ion etching, completing the formation of individually addressable microdisk LEDs in free-standing form.

The electrode alignment and integrity of crystalline interfaces within the microdisk LEDs were confirmed using SEM and scanning transmission electron microscopy (STEM). Figure 2b shows that each metal strip, whether on the top or bottom, continuously covered only a single row or column of microdisks, indicating that each microdisk can be uniquely addressed. High-angle annular dark-field STEM images of a cross-sectioned microdisk LED, displayed in Fig. 2c, further confirms the formation of conformal and high-quality interfaces between the 


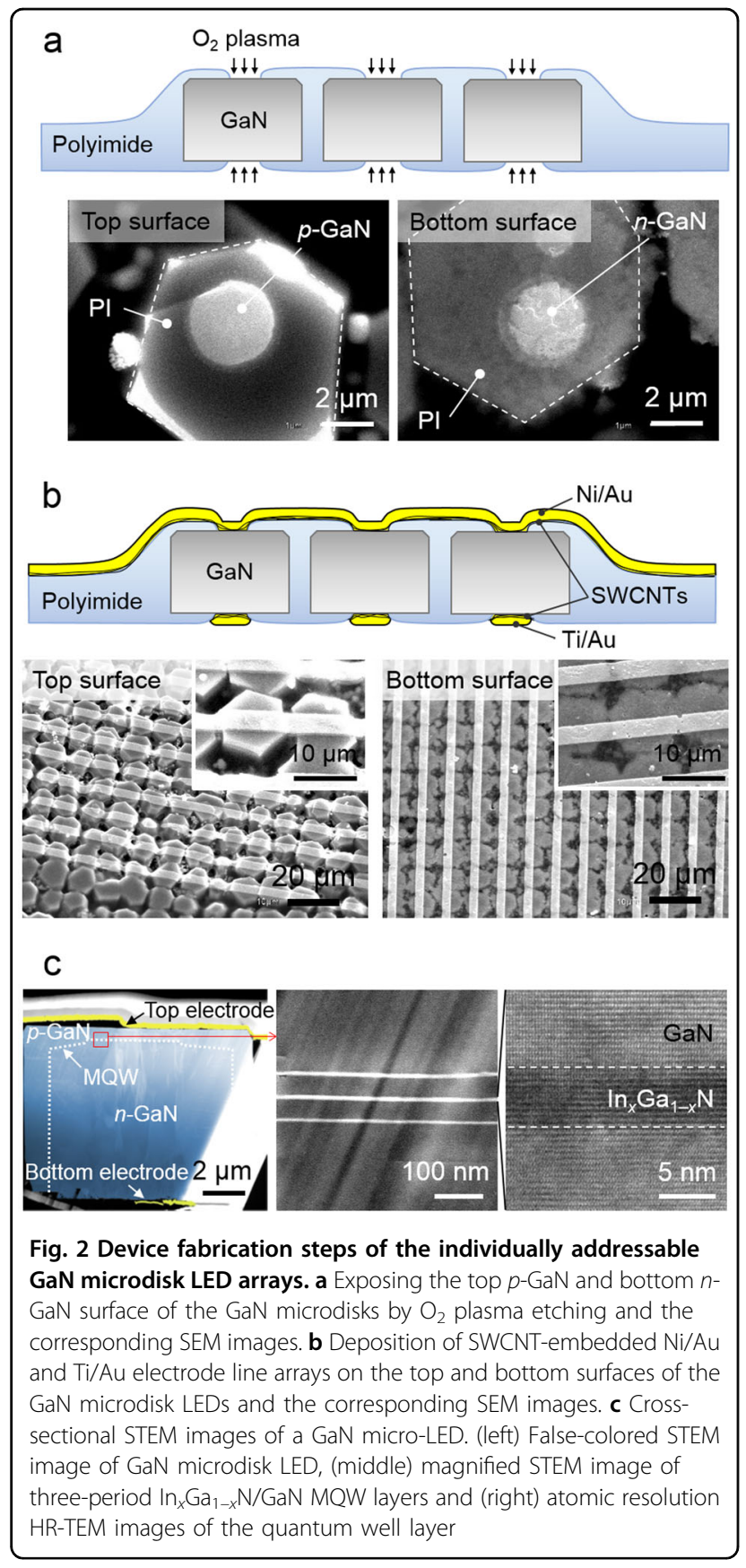

metal electrodes and GaN. The yellow and blue regions denote the $\mathrm{Au}$ and $\mathrm{GaN}$ regions, respectively. Both top and bottom electrodes shared direct contact with the top and bottom GaN surfaces. High-magnification images of the $\mathrm{p}-\mathrm{n}$ homojunction further reveal the presence of three bright lines with a uniform thickness of $7.0 \pm 0.5 \mathrm{~nm}$ corresponding to $\mathrm{In}_{x} \mathrm{Ga}_{1-x} \mathrm{~N}$ quantum well layers. Atomic resolution images of the quantum well indeed show that the interfaces were uniform and clean. Furthermore, no signs of structural defects, such as dislocations or stacking faults, were observed near the vicinity of the MQW interface, highlighting the high-quality crystallinity of the inorganic materials.

\section{Device characteristics of free-standing and ultrathin, individually addressable microdisk LED array}

Light emission from the individual microdisk LEDs in free-standing and ultrathin form was investigated by characterizing the EL properties. The EL from each microdisk LED was measured by electrically contacting the respective pair of top and bottom electrode pads and applying a forward bias voltage of $15 \mathrm{~V}$. The optical microscope image obtained from a representative microdisk LED demonstrated a bright emission localized at its respective position, as shown in Fig. 3a. Figure 3b demonstrates that the EL emission can be freely translated to a new position by selecting the respective pair of top and bottom electrode pads. As each microdisk LED can be considered a pixel spaced apart by a $10 \mu \mathrm{m}$ pitch, we found the pixel resolution of the array to be as high as 2500 pixels per inch (ppi). This is one to two orders of magnitude higher than the conventional display pixel density of 70-300 ppi, suggesting that microdisk LED arrays hold great promise for ultrahigh resolution applications.

It was expected that the unique LED array structure consisting of discrete microdisk arrays embedded in a flexible polymer layer should allow the device to tolerate extreme bending conditions. Accordingly, the stability of the electrical and EL characteristics under various bending radii was investigated. Figure $3 \mathrm{c}$ shows photographs and corresponding current-voltage (I-V) curves from the array subjected to bending radii of 10,3 , and $1 \mathrm{~mm}$. The I-V curves obtained at different bending radii exhibited almost identical behavior (see Fig. 3c), suggesting that no serious mechanical stress, damage, or fracture occurred at the electrodes or the junctions between the $\mathrm{GaN}$ microdisks. The durability of the electrodes under harsh bending conditions can be attributed to the presence of SWCNTs embedded in the metal as they provided mechanical support to the electrodes (see Fig. S2 in Supplement) ${ }^{23-26}$.

The EL spectra and power of an individual microdisk LED were also measured at various bias voltages in the range of 5-19 V. As shown in Fig. 4a, a dominant EL peak started to appear at $456 \mathrm{~nm}$ with a full width at half maximum (FWHM) of $80 \mathrm{~nm}$. As the applied bias voltage increased to $19 \mathrm{~V}$, the EL peak blueshifted toward $440 \mathrm{~nm}$ with a reduced FWHM of $22 \mathrm{~nm}$. This EL peak is roughly twice as sharp as that of ultrathin blue polymer LEDs exhibiting an FWHM of $50 \mathrm{~nm}^{11}$. Such results indicate that the color saturation of the microdisk LED was superior to that of the polymer LED. In addition, we observed a slight variation in the EL colors among the microdisk LEDs in the same array, where the EL peak positions of different LEDs were observed between 440 
a

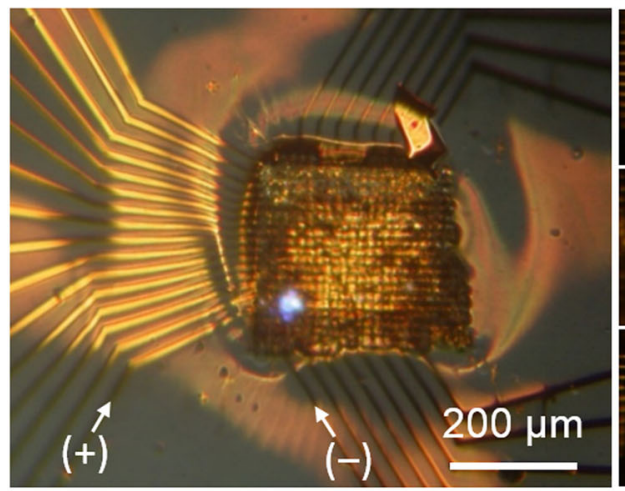

C

\section{Bending radius,}

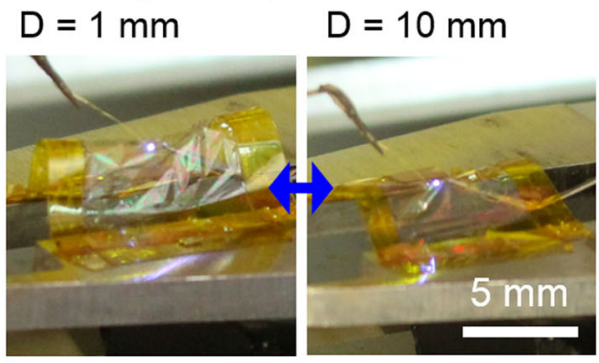

b
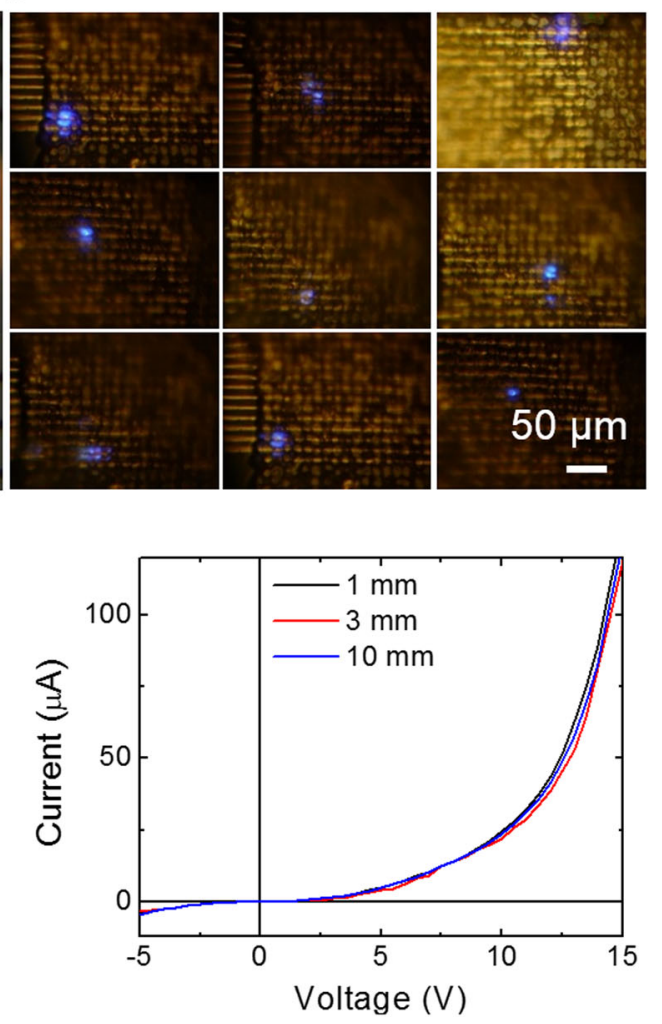

Fig. 3 EL images of the individually addressable microdisk LED microarrays. a EL image of a single microdisk LED at $15 \mathrm{~V}$. $\mathbf{b}$ EL image of the microdisk LEDs driven with different probing positions. c Photograph of the free-standing device under different bending conditions and the corresponding $\mathrm{I}-\mathrm{V}$ characteristics

and $450 \mathrm{~nm}$ with average and standard deviation of 453 and $3 \mathrm{~nm}$, respectively. We believe that the variation in EL colors was due to small difference in the sizes of the microdisk LEDs that caused a slight variation in the $\mathrm{In}_{x} \mathrm{Ga}_{1-x} \mathrm{~N} / \mathrm{GaN}$ multiple quantum well compositions ${ }^{27}$ and that the EL color uniformity could be improved by further optimizing the growth uniformity of the GaN microdisk. Figure $4 \mathrm{~b}$ shows the integrated EL intensity as a function of bias voltage. The EL intensity increased linearly with increasing current, demonstrating that the light emission resulted from carrier injection through the $\mathrm{p}-\mathrm{n}$ junction of the microdisk LED. The turn-on voltage of the microdisk LED was observed near $5 \mathrm{~V}$, at which point we started to observe light emission from the LED, and the EL output power per unit area increased up to $0.25 \mathrm{nW} / \mu \mathrm{m}^{2}$. In addition, we observed that the microdisk LEDs were found to operate reliably in free-standing form under continuous mode (see Fig. S3).

To further understand the EL properties of the multifaceted microdisk LED, the current spreading characteristics inside the LED were calculated using a finite element method package. A simple cylindrical $\mathrm{p}-\mathrm{n}$ junction GaN microdisk was used to model the device, and a forward bias of $10 \mathrm{~V}$ was applied to the device. Figure 4c shows the cross-sectional view of the device, including the calculated results, where the streamlines represent the current flow, and the intensity of the red color represents the current density. The yellow areas indicate ohmic metal electrodes around the microdisk. The current density was strong near the central $z$-axis of the device and became weaker for positions radially distant from the axis. In addition, the current flow intersected the horizontal $\mathrm{p}-\mathrm{n}$ junction more readily than the vertical $\mathrm{p}-\mathrm{n}$ junction. This indicates that charge recombination and thus light emission mostly occurred from the central region of the horizontal $\mathrm{p}-\mathrm{n}$ junction.

Aided by the above results, we further characterized the far-field light output pattern generated by a single microdisk LED within an array. Using finite difference time domain simulations, a collection of incoherent dipoles was introduced along the horizontal $\mathrm{p}-\mathrm{n}$ junction of a single microdisk to model the light emission in the microcavity. Figure $4 \mathrm{~d}$ describes the normalized far-field light distribution in polar coordinates. Careful inspection 

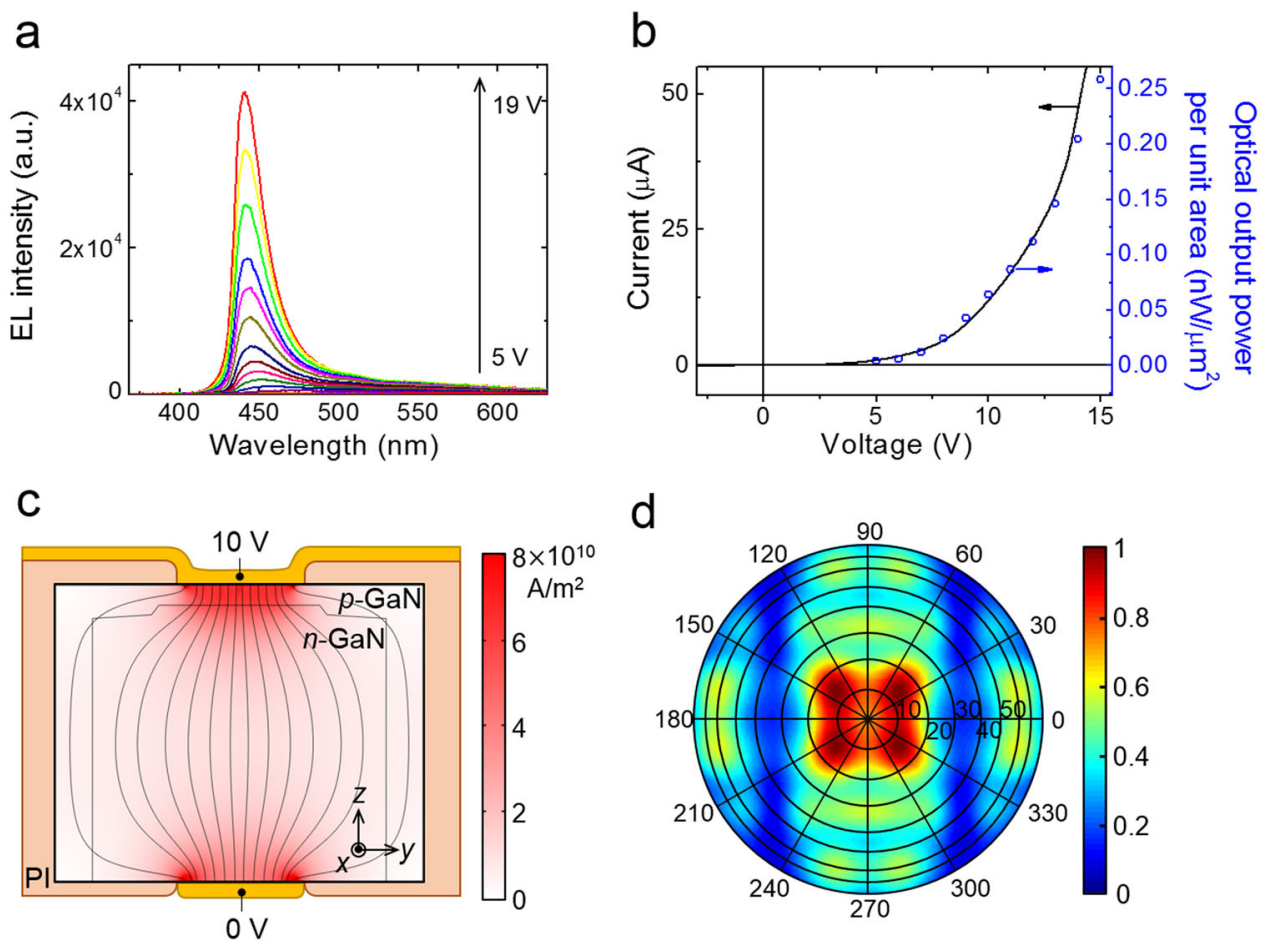

Fig. 4 a EL spectra of a single GaN microdisk LED within the array under increasing bias voltages. $\mathbf{b}$ I-V curve and voltage-dependent EL intensity of a single GaN microdisk LED within the array. c Current spreading characteristics inside the GaN microdisk LED under an external bias voltage of $10 \mathrm{~V}$. $\mathbf{d}$ Far-field electric field pattern generated by a single microdisk within an array

of the distribution near the center revealed four bright lobes sharing a square symmetry. Furthermore, the intensity is reduced at the center of the pattern and along the central $y$-axis $(x=0)$. This is attributed to the fact that the top metal electrode is aligned along the $y$-axis, blocking light emission from incoherent dipoles positioned directly underneath it. However, one can see that the bright intensity was confined to a narrow half angle of $20^{\circ}$. Calculations of the integrated far-field intensity show that $\sim 15 \%$ of the total power was emitted into the $20^{\circ}$ cone, indicating that the microdisks emit light the strongest in the forward direction.

\section{Conclusion}

We demonstrated the fabrication and characteristics of an individually addressable GaN microdisk LED array in free-standing and ultrathin form. The excellent mechanical flexibility of the device was largely made possible by the ability to grow and transfer inorganic microdisk arrays to flexible substrates using graphene microdots as growth and release sites. Stable electrical and optoelectronic performances were observed under extreme bending conditions, and the device was found to operate reliably in free-standing form without the presence of a heat dissipating substrate. Each microdisk LED constituting an array of ultrahigh resolution of 2500ppi was found to be uniquely addressable. The superior optoelectronic characteristics derived from the high crystallinity of the microdisks and excellent mechanical flexibility enabled by the free-standing and ultrathin form allow the microdisk LED array to be used as a flexible and high-performance device in advanced wearable, medical, and implantable applications.

\section{Acknowledgements}

This work was financially supported by the Samsung Research Funding Center of Samsung Electronics (SRFC-TA1803-02(0417-20180116)).

\section{Author details}

${ }^{1}$ Department of Physics and Astronomy, Institute of Applied Physics, and Research Institute of Advanced Materials, Seoul National University, Seoul 08826, Korea. ${ }^{2}$ Department of Chemistry and Nanoscience, Ewha Womans University, Seoul 03760, Korea. ${ }^{3}$ Department of Material Science and Engineering, Seoul National University, Seoul 08826, Korea

\section{Conflict of interest}

The authors declare that they have no conflict of interest.

\section{Publisher's note}

Springer Nature remains neutral with regard to jurisdictional claims in published maps and institutional affiliations.

Supplementary information is available for this paper at https://doi.org/ 10.1038/s41427-019-0137-7. 
Received: 7 January 2019 Revised: 17 April 2019 Accepted: 19 May 2019. Published online: 19 July 2019

\section{References}

1. Templier, F. GaN-based emissive microdisplays: a very promising technology for compact, ultra-high brightness display systems. J. Soc. Inf. Disp. 24, 669-675 (2016).

2. Rogers, J. A., Someya, T. \& Huang, Y. Materials and mechanics for stretchable electronics. Science 327, 1603-1607 (2010).

3. Herrnsdorf, J. et al. Active-matrix GaN micro light-emitting diode display with unprecedented brightness. IEEE Trans. Electron Devices 62, 1918-1925 (2015).

4. Kim, Y. et al. Remote epitaxy through graphene enables two-dimensional material-based layer transfer. Nature 544, 340 (2017)

5. Kim, T.-i et al. Injectable, cellular-scale optoelectronics with applications for wireless optogenetics. Science 340, 211-216 (2013).

6. Ponce, F. \& Bour, D. Nitride-based semiconductors for blue and green lightemitting devices. Nature 386, 351-359 (1997).

7. Nakamura, S. The roles of structural imperfections in InGaN-based blue lightemitting diodes and laser diodes. Science 281, 956-961 (1998).

8. Strite, S. \& Morkoç, H. GaN, AlN, and InN: a review. J. Vac. Sci. Technol. B 10, 1237-1266 (1992).

9. Pearton, S., Norton, D., I, K., Heo, Y. \& Steiner, T. Recent progress in processing and properties of ZnO. Prog. Mater. Sci. 50, 293-340 (2005).

10. Akasaki, I. \& Amano, H. Breakthroughs in improving crystal quality of GaN and invention of the p-n junction blue-light-emitting diode. Jpn. J. Appl. Phys. 45 (12R), 9001 (2006)

11. Yokota, T. et al. Ultraflexible organic photonic skin. Sci. Adv. 2(4), e1501856 (2016).

12. Forrest, S. R. The path to ubiquitous and low-cost organic electronic appliances on plastic. Nature 428, 911 (2004).
13. Hammock, M. L., Chortos, A., Tee, B. C. K., Tok, J. B. H. \& Bao, Z. 25th anniversary article: the evolution of electronic skin (e-skin): a brief history, design considerations, and recent progress. Adv. Mater. 25, 5997-6038 (2013).

14. Ganii, M. et al. Development and Translation of PEDOT: PSS Microelectrodes for Intraoperative Monitoring. Adv. Funct. Mater. 28, 1700232 (2018).

15. Kim, D. H., Xiao, J., Song, J., Huang, Y. \& Rogers, J. A. Stretchable, curvilinear electronics based on inorganic materials. Adv. Mater. 22, 2108-2124 (2010).

16. Fan, Z. et al. Three-dimensional nanopillar-array photovoltaics on low-cost and flexible substrates. Nat. Mater. 8, 648 (2009).

17. Park, S.I. et al. Printed assemblies of inorganic light-emitting diodes for deformable and semitransparent displays. Science 325, 977-981 (2009).

18. Lee, H. E. et al. Monolithic flexible vertical GaN light-emitting diodes for a transparent wireless brain optical stimulator. Adv. Mater. 30, 1800649 (2018).

19. Guan, N. et al. Flexible white light emitting diodes based on nitride nanowires and nanophosphors. ACS photonics 3, 597-603 (2016).

20. Chung, K., Lee, C.-H. \& Yi, G.-C. Transferable GaN layers grown on ZnO-coated graphene layers for optoelectronic devices. Science 330, 655-657 (2010).

21. Lee, C. H. et al. Flexible inorganic nanostructure light-emitting diodes fabricated on graphene films. Adv. Mater. 23, 4614-4619 (2011).

22. Chung, K. et al. Flexible GaN light-emitting diodes using GaN microdisks epitaxial laterally overgrown on graphene dots. Adv. Mater. 28, 7688-7694 (2016).

23. Cao, Q. et al. Medium-scale carbon nanotube thin-film integrated circuits on flexible plastic substrates. Nature 454, 495 (2008).

24. Hong, S. \& Myung, S. Nanotube electronics: a flexible approach to mobility. Nat. Nanotechnol. 2, 207 (2007).

25. Tsai, J. T. H. \& Hwang, H.L. Carbon nanotube reinforced conductors for flexible electronics. J. Disp. Technol. 5, 232-235 (2009).

26. Cox, N. D. et al. Free-standing silver/carbon nanotube metal matrix composite thin films. J. Mater. Sci. 51, 10935-10942 (2016).

27. Tchoe, Y. et al. Variable-color light-emitting diodes using GaN microdonut arrays. Adv. Mater. 26, 3019-3023 (2014). 